\title{
Chaperonopathies and Chaperonotherapy. Hsp60 as Therapeutic Target in Cancer: Potential Benefits and Risks
}

\author{
Francesco Cappello ${ }^{1,2}$, Francesca Angileri ${ }^{1,2}$, Everly Conway de Macario ${ }^{3}$ and Alberto J. L. Macario ${ }^{2,3, *}$
}

${ }^{I}$ Dipartimento di Biomedicina Sperimentale e Neuroscienze Cliniche, Sezione di Anatomia Umana, University of Palermo, Palermo, Italy; ${ }^{2}$ Istituto Euro-Mediterraneo di Scienza e Tecnologia (IEMEST), Palermo, Italy; ${ }^{3}$ Department of Microbiology and Immunology, School of Medicine, University of Maryland at Baltimore, and IMET, Baltimore, MD, USA

\begin{abstract}
In this minireview we focus on Hsp60 as a target for anticancer therapy. We discuss the new concepts of chaperonopathies and chaperonotherapy and present information on Hsp60 localization in the cell membrane of human tumor cells. We describe novel mechanisms for Hsp60 reaching the extracellular environment that involve membrane-associated stages, as well as data on anti-Hsp60 antibodies found in human sera, both in normal subjects and patients affected by autoimmune diseases. Finally, we discuss possible therapeutic applications of anti-Hsp60 antibodies in cancer treatment, evaluating also side effects on non-tumor cells. In conclusion, the way for investigating Hsp60-targeted anti-tumor therapy is open, at least for those tumors that express Hsp60 on its surface and/or secrete it outside the cell, as is the search for the molecular mechanisms involved in Hsp60 translocation from cytosol to cell membrane: elucidation of this mechanism will greatly facilitate the optimization of chaperonotherapy centered on Hsp60 with anti-tumor efficacy and minimal side effects.
\end{abstract}

Keywords: Hsp60, Cpn60, HSPD1, plasma membrane, antibodies, autoantibodies, antitumor immunotherapy.

\section{CHAPERONES AND PATHOLOGY: THE CHAPERO- NOPATHIES}

Molecular chaperones (chaperones in short) constitute a large group of proteins with a variety of functions, some of which are crucial for organismal survival [1]. Chaperones have been described at first as players in a number of intracellular pathways pertaining to protein quality control such as protein folding, refolding and degradation after irreversible misfolding, and protein translocation but, as of late, other functions unrelated to protein homeostasis have been discovered, for instance apoptosis regulation and cell signaling [2]. Moreover, chaperones have been shown to participate in antistress mechanism to protect cells and organisms against stressors and stress and its consequences [3, 4]. Finally and most interestingly, has been the demonstration that chaperones have active participation in extracellular events, as in cell-to-cell cross talk and immune system regulation [5-8].

Nowadays, chaperones are seen as indispensable for cell and tissue homeostasis in all organisms, including humans [8, 9]. However, chaperones have also been recognized to play a pathogenic role in a number of diseases, now called chaperonopathies, because they are qualitatively and/or quantitatively altered or function to favor disease rather than the contrary $[9,10]$. It is likely that awareness of the role of chaperones in the etiopathogenesis of many diseases will change the nosography in all health related disciplines, basic and applied. Moreover, the possibility to cure a chaperonopathy caused by a defective or malfunctioning chaperone by tailored chaperonotherapy will contribute to improving life quality in a number of pathologic conditions $[11,12]$.

In this article, we review recent findings pertaining to the potential of the chaperone Hsp60, namely the Heat Shock Protein 60 $\mathrm{kDa}$, also called chaperonin 60 (Cpn60) or HSPD1 protein [13], in cancer management.

\footnotetext{
*Address correspondence to this author at the Department of Microbiology and Immunology, School of Medicine, University of Maryland at Baltimore, and IMET, Baltimore, MD, USA; Columbus Center, 701 East Pratt Street, Baltimore, MD 21202, USA; Tel/Fax: 240-631-9594;

Tel: 410-234-8886; Fax: 410-234-8896;

E-mail: Ajlmacario@som.umaryland.edu
}

\section{CHAPERONES IN THERAPEUTICS: CHAPERONOTHER- APY}

The field of chaperonotherapy is mostly based on the cytoprotective effects that molecular chaperones have and that confer stress resistance to cells [11]. However, chaperonotherapy has in fact two opposite facets. That based on the cytoprotective action of chaperones, just mentioned, and another, based on the fact that some chaperones can be pathogenic. In this latter case, a normal chaperone promotes disease rather than the contrary. In these conditions, chaperonopathies by mistake or collaborationism [10], the therapeutic action should be aimed at blocking or eliminating the mistaken chaperone. Illustrative examples of chaperonopathies by mistake or collaborationism are some types of tumors in which a chaperone, e.g., Hsp70 or Hsp90 (or both), is essential for tumor growth and dissemination. In these situations, antichaperone agents would be the choice for treatment. Along these lines, there are many reports on chaperonotherapy at the experimental level and we cite here only very few of the many illustrative examples available: they pertain to Hsp90 inhibitors and to the administration of chaperones and to gene replacement for managing experimental conditions mimicking human cancer, autoimmune inflammation, and retinal and neurodegenerative disorders [14-21].

In this work, we present the potential of Hsp60 for chaperonotherapy but, before, we will discuss some critical aspects of this chaperonin's biology in humans and the occurrence of anti-Hsp60 antibodies. We will outline the travels of Hsp60 in the body and the impact of them on the immune system and the potential role of antiHsp60 antibodies in therapeutics.

\section{HSP60 AND ITS ODYSSEY FROM THE INTRACELLULAR ENVIRONMENT TO DISTANT TARGETS}

Most of the information available about functions of Hsp60 has been obtained by studies on its bacterial counterpart, GroEL [22, 23]. Hsp60, like several other groups of chaperones, is evolutionarily conserved [24, 25]. Both Hsp60 and GroEL are involved in protein folding, working in combination with a co-chaperone, Hsp10 (GroES in bacteria) [26]. In eukaryotes, the Hsp60/Hsp10 molecular machinery is localized inside the mitochondria and is crucial for maintaining the mitochondrial respiratory-chain integrity and functionality and, consequently, cell survival $[27,28]$. This is valid not only in normal but also in tumor cells, in which Hsp60 levels are 
frequently increased especially in extramitochondrial sites ((Fig. 1) and Table 1) [29].

In addition, during evolution Hsp60 acquired functions other than chaperoning in the classical sense of maintaining protein homeostasis, such as regulation of glycogenesis and apoptosis [16, 3032]. For example, Hsp60 can exit mitochondria after cell stress and activate the caspase pathway by inducing caspase-3 cleavage. In contrast, when Hsp60 accumulates in the cytosol without mitochondrial release (a frequent condition during carcinogenesis) it has the opposite effect, i.e., it inhibits caspase-3 cleavage [31]. The latter may be a mechanism that operates for at least some tumors for cell proliferation during carcinogenesis. In this case, Hsp60 seems to help the neoplasm rather than to protect the host and, therefore, these forms of cancer have been classified as chaperonopathies "by mistake" or "collaborationism" [10].

In certain conditions, cytosolic Hsp60 translocates to cell membrane $[22,23]$. In order to explain this movement of the chaperonin, various molecular mechanisms, including epigenetic or posttranslational modifications, have been proposed, but none has yet been definitively established. Nonetheless, this translocation event can have important consequences because circulating antibodies could bind to the Hsp60 present on the cell membrane and thereby affect cell viability. In what pertains to tumor cells, the translocation event with localization of Hsp60 on the cell membrane can be considered a major tenet to develop novel anti-tumor therapy strategies for those tumors that do show this characteristic, mimicking what happens with Hsp70 [5, 12].

Hsp60 could be the target of specific monoclonal antibodies or antitumor vaccination protocols. However, it is not yet established for how long Hsp60 remains on the tumor-cell membrane. This time-period could, indeed, be very short because lipid rafts - specialized membrane microdomains - can remove and internalize membrane Hsp60 into multivesicular bodies, which in turn generate
Table 1. Comparison of Subcellular Distribution of Hsp60 in Normal and Tumor cells ${ }^{\mathrm{a}}$

\begin{tabular}{|c|c|c|}
\hline \multirow{2}{*}{ Cell Compartment } & \multicolumn{2}{|c|}{ Cell Type } \\
\cline { 2 - 3 } & Normal & Tumor \\
\hline \hline Mitochondria & +++ & +++ \\
\hline Cytosol & + & +++ \\
\hline Membrane & $-/+$ & ++ \\
\hline Vesicles & $-/+$ & ++ \\
\hline Endoplasmic Reticulum / Golgi & $-/+$ & + \\
\hline
\end{tabular}

aSemiquantitative evaluation of Hsp60 in the compartments of normal and tumor cells, using transmission electron microscopy-immunogold with specific anti-Hsp60 antibodies (for methodology see $[33,50]$, and references therein). The results are expressed in an arbitrary scale from $-=$ Hsp60 undetectable, to $+++=$ Hsp60 abundantly present. The tumor cells studied were NCI-H292 (human mucoepidermoid bronchial carcinoma), A549 (human lung adenocarcinoma) and K562 (human erythroleukemia) cell lines and were compared to 16HBE (human bronchial epithelium) and primary cultures of human dermal fibroblasts. Evaluation was made by two electron microscopists and the data presented reflect the mean of the results for each cell type and compartment. Personal data unpublished.

Hsp60-loaded exosomes [33]. The latter are microvesicles involved in cell-to-cell cross talk and immune system regulation, as well as in tumor progression [34]. We have found that the tumor cells we tested actively secrete Hsp60 via exosomes (Fig. 2). In addition, Hsp60 can also be found in its free/soluble form in circulation [29], which suggests that the Golgi's apparatus is also involved in its secretion from tumor cells. (Fig. 3) depicts a working model we have proposed for the "Odyssey" of Hsp60 when it moves from cytosol of tumor cells to extracellular space and then to circulation.
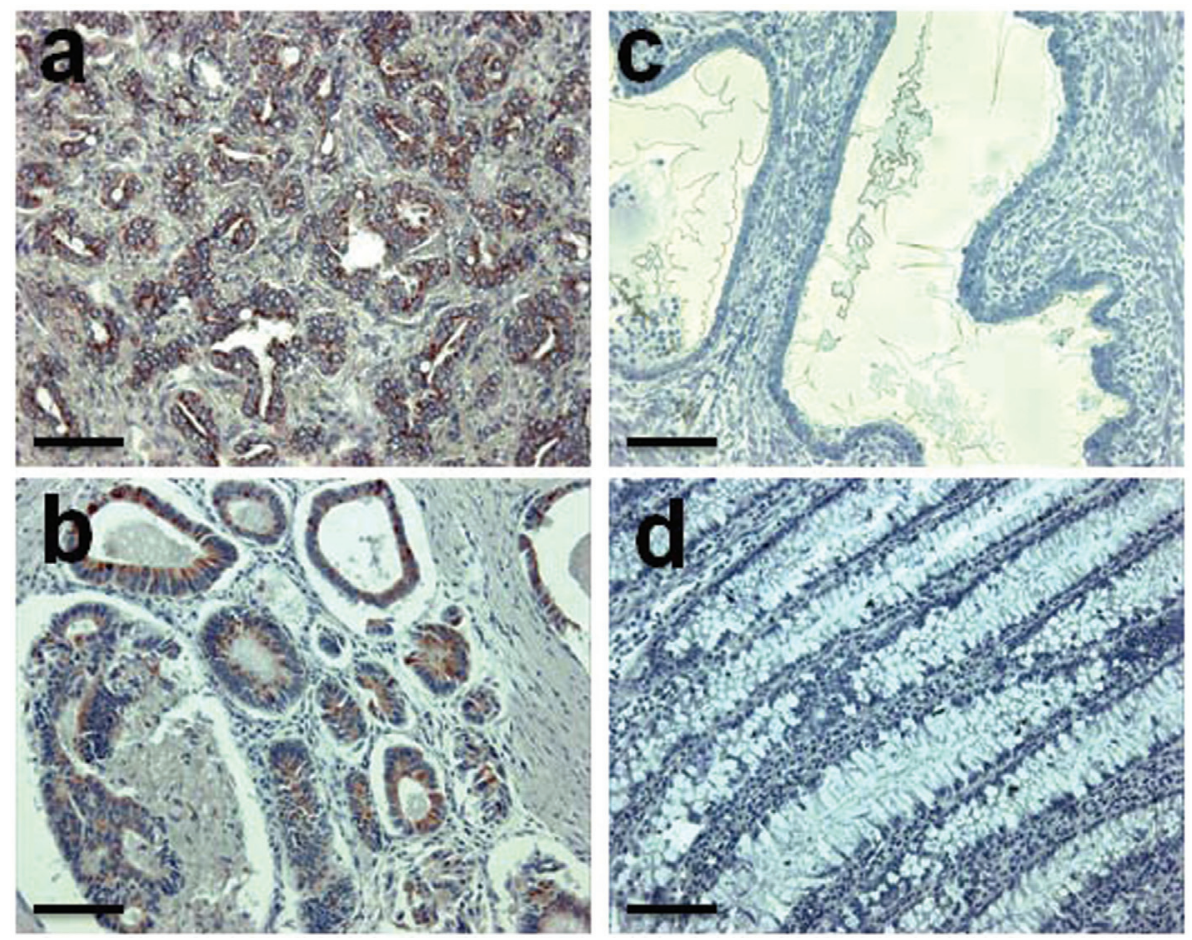

Fig. (1). Hsp60 levels in tumor cells are frequently higher than in the normal tissue counterpart.

The images show Hsp60 (brown color, darkest areas in black and white reproductions) detected by immunohistochemistry with specific anti-Hsp60 antibodies in carcinoma of prostate (a) and colon (b). Normal prostate tissue and colonic mucosa are shown in (c) and (d), respectively, in which Hsp60 is almost undetectable (no brown color). Bar, 200 microns. For details about immunohistochemical detection of Hsp60 in tissues, see [44]. (The color version of the figure is available in the electronic copy of the article). 

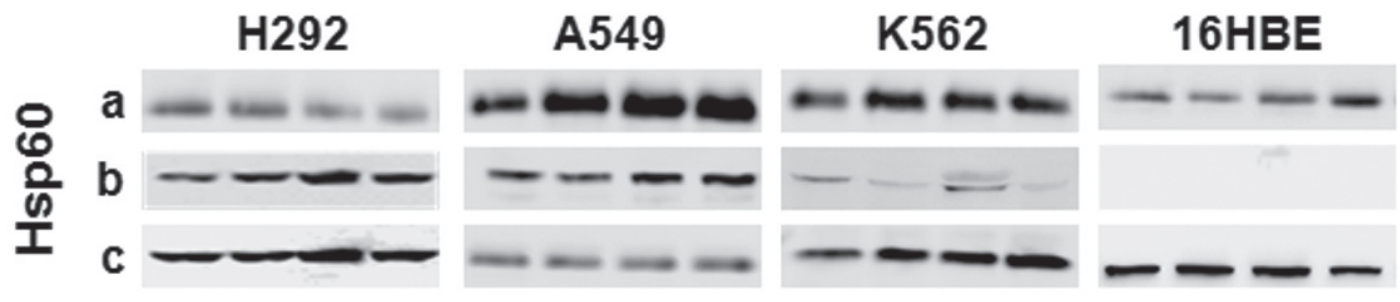

Fig. (2). Hsp60 is released by tumor (H292, A549, and K562) but not by non-tumor (16BHE) cells into the extracellular environment via exosomes. Demonstration of Hsp60 by Western blotting in (a) immunoprecipitates from the conditioned media; (b) exosomes purified from the conditioned media; (c) whole-cell lysates. Each set of four Western Blot lanes represents four separate experiments. The data showed that while all cells had Hsp60 inside them (c) and all released Hsp60 into the extracellular space (a), only tumor cells did it via exosomes (b). These results were confirmed in various ways, demonstrating that the process was an active secretion by healthy, viable, tumor cells, excluding passive release by dead and disrupted or damaged cells [33]. Reproduced, modified with permission from reference [33].

\section{TUMOR CELL}

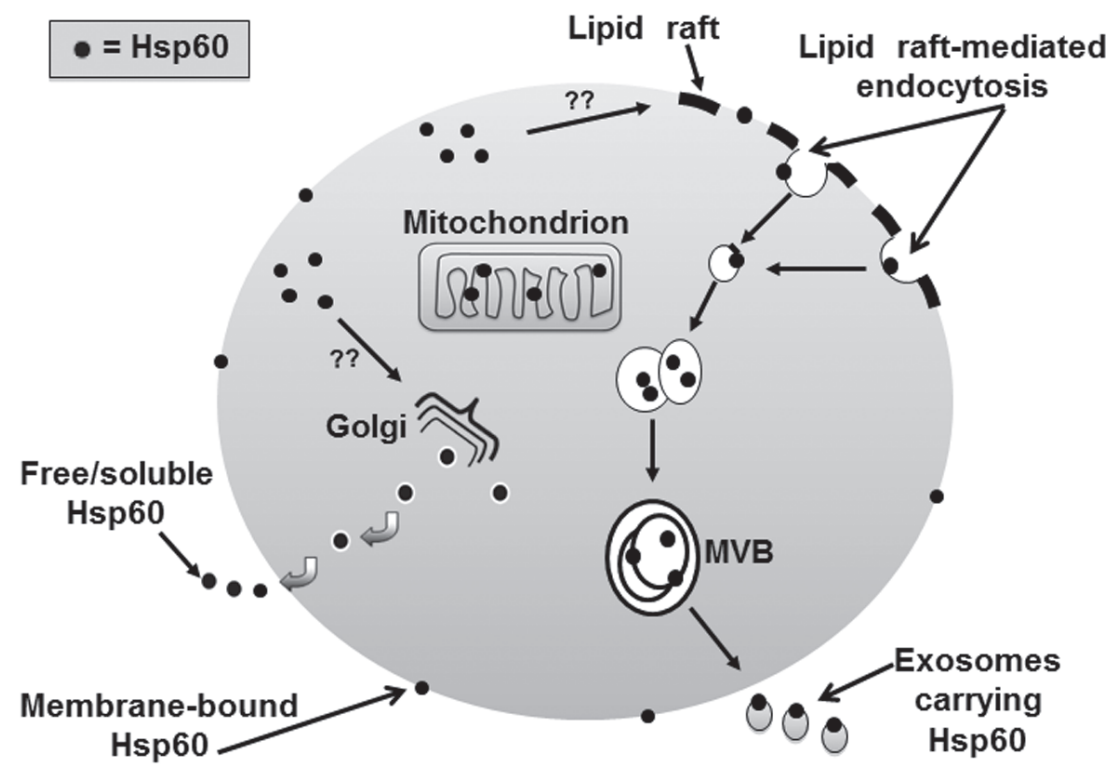

MVB = multivesicular bodies

Fig. (3). Itineraries of Hsp60 in tumor cells.

This figure summarizes, schematically, the conclusions derived from experimental observations, focusing on the various locations demonstrated for Hsp60 in cells. Hsp60 (black dots) in normal cells localizes mainly in mitochondria but in tumor cells it is found also in the cytoplasm and the cell membrane. In it, lipid rafts internalize Hsp60 toward multivesicular bodies (MVB) from where it is secreted via exosomes. Other data suggest that the Golgi may also participate in Hsp60 secretion (under investigation). Details are still missing for some of the intermediary steps, as illustrated by questions marks.

Some steps of these pathways are not yet fully elucidated and are currently under investigation.

\section{ANTI-HSP60 ANTIBODIES: DANGER OR SAFETY?}

An increase in membrane Hsp60 and the presence of circulating Hsp60 are considered danger signals for the immune system in as much as they may lead to activation of immune cells [29, 35]. Information about Hsp60 and activation of innate and adaptive immunities has been published and discussed elsewhere [35-37]. Here we concentrate on information about the specific humoral (antibody-mediated) immune response induced by Hsp60.

Human anti-Hsp60 antibodies have been found in normal populations [38] and in subjects with autoimmune diseases [39]. Hence, it has been postulated that an inappropriate immune reaction to Hsp60 contributes to autoimmune disease development [38].
Due to the high degree of evolutionary conservation from microbial GroEL to human Hsp60, it is likely that some autoimmune conditions are initiated by bacterial infections that release the chaperonin into the circulation with the resulting production of antiHsp60 antibodies. These antibacterial Hsp60 antibodies will crossreact with the human chaperonin [35]. It is a difficult challenge to distinguish between the latter situation and those autoimmune conditions primarily caused by the self, human Hsp60 with autoantibodies produced against it $a b$ initio. Whatever the mechanism that initiates anti-Hsp60 antibody production, one has to realize that these autoantibodies can cause not only autoimmune diseases but also they can participate in the destruction of tumor cells expressing Hsp60 on their surface [35].

The antibody-antigen reaction can occur at the cell surface and also in the blood, generating tumor-cell lysis and immunocom- 
plexes, respectively [35]. In turn, the former induces cell death, while the latter can lead to the formation of immunoprecipitates in the renal glomeruli for instance [35]. We have previously postulated that if Hsp60 occurs on the surface of malignant cells, as already ascertained for some types of cancer, anti-Hsp60 antibodies have the potential ability to destroy the tumor [29, 35, 40]. Our hypothesis has been recently supported by observations on multiple myelomas [41]. It was reported that immunoglobulins (Ig) from myeloma patients after allogenic stem-cell transplantation recognized Hsp60 aberrantly displayed on the surface of primary myeloma cells, whereas Ig from a control group of healthy donors did not. The authors confirmed that Hsp60 becomes accessible to the immune system after aberrant membrane exposure and, naturally, postulated that a humoral anti-chaperonin response may contribute to the more favorable prognosis shown by these patients after transplantation.

\section{POSSIBLE APPLICATIONS OF, AND CAUTIONARY COMMENTS ON, HSP60-TARGETED ANTICANCER THERAPY}

The few publications discussed above on involvement of Hsp60 in human carcinogenesis constitute a minimal but representative sample of what has been published on this matter and we refer to other reviews for a more comprehensive account [29, 36, 40, 42]. The conclusion is that information in the literature from our laboratories and those of others unmistakably justifies considering Hsp60 among candidates for anti-cancer immunotherapy.
Hsp60 is increased in many forms of cancers, although in others its levels decrease [29]. When increased, Hsp60 may localize in the tumor-cell membrane as Hsp70 [43]. When exposed on the cell membrane, Hsps become accessible to circulating anti-Hsp antibodies [41]. The presence of Hsp60 on the cell surface offers practical advantages in medicine. For example, from the diagnostic standpoint, immunofluorescence and immunocyto- and immunohistochemistry with the proper antibodies are useful methods for the pathologist to detect Hsp60 on the cell surface [44]. In addition, some studies open the possibility for exploring the use of either free or exosome-carried Hsp60 for vaccination against cancer $[45,46]$.

If Hsp60 favors cancer growth, chaperonotherapy will consist of blocking the chaperonin by whatever method works without serious side effects. If the chaperonin offers a target on the tumorcell surface for specific antibodies that could initiate cell lysis, the use of these antibodies should then be part of anti-tumor chaperonotherapy. In any case, it is obvious that the road to the development of antitumor strategies and reagents centered on Hsp60 is open and very inviting.

However, the probability of unwanted side effects of this form of therapy must be taken into consideration. Physical and mechanical stress, as well as aging, may cause Hsp60 dislocation onto the cell membrane. For example, hypertension can induce Hsp60 exposure on the surface of endothelial cells [47]. Other cells in which Hsp60 has been localized onto the plasma membrane are cardiomyocytes during heart failure [48] and senescent platelets [49]. Importantly, a small quantity of membrane Hsp60 has been found

\section{THE BENEFIT}

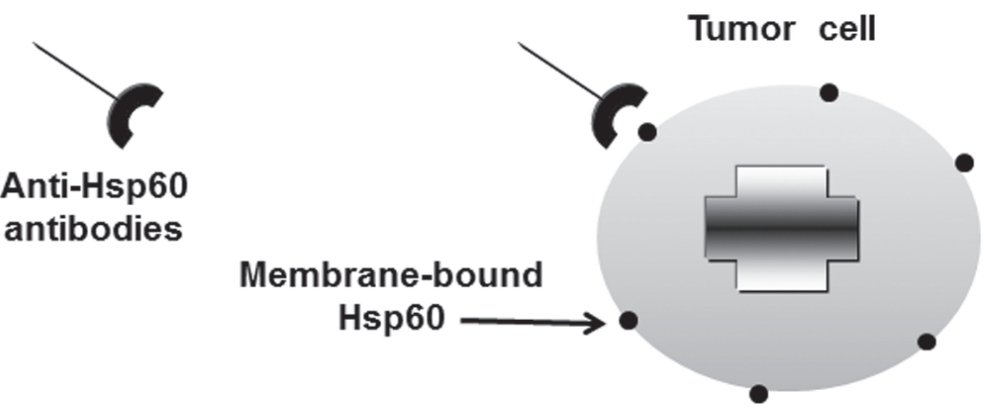

\section{THE RISKS}
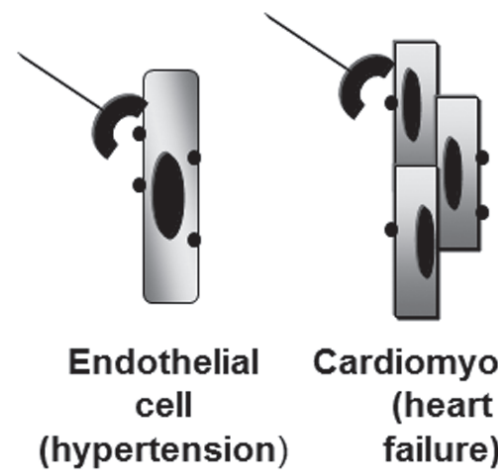

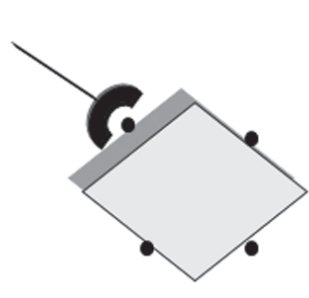

\section{Platelet (senescent)}

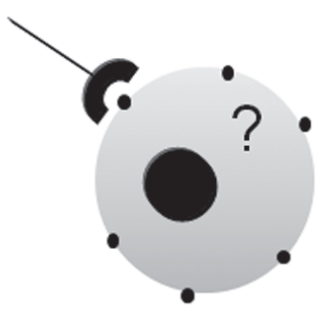

$$
\begin{gathered}
\text { Other cells } \\
\text { (under } \\
\text { investigation) }
\end{gathered}
$$

Fig. (4). Benefits and possible risks of antitumor therapy by anti-Hsp60 antibodies.

Anti-Hsp60 antibodies are potentially able to destroy tumor cells that present aberrant Hsp60 on the cell membrane and this offers an opportunity for developing anti-cancer immunotherapy centered on the chaperonin. However, other cytotypes (e.g., endothelial cells, cardiomyocytes, and platelets), not just tumor cells, in certain conditions may also expose Hsp60 on the cell membrane, a situation that would put non-tumor cells at risk of lysis induced by anti-Hsp60 antibodies. Evaluation of this risk and the possible advantages of Hsp60 chaperonotherapy should be preferred objectives of cancer research in the upcoming years in order to prepare the road for clinical trials. 
also in normal mammal cells, including human fibroblasts [50]. Hence, cytotoxicity caused by anti-Hsp60 antibodies can potentially affect non-tumor but altered, and even normal, cells in addition to the cancer cells and, thus, generate severe adverse complications (Fig. 4). In addition, the presence of circulating Hsp60 may determine the formation of immunocomplexes that might deposit in the glomerular membrane, generating glomerulonephritis.

Another potential drawback of anti-Hsp60 antibodies as antitumor agents is that internalization of Hsp60 via the lipid rafts pathway may occur at a rapid rate, reducing drastically the time of Hsp60 availability on the tumor cell membrane and, thus, limiting the efficacy of anti-Hsp60 immunotherapy. Another inconveniency to explore is that the removal of tumor cells exposing Hsp60 on their cell membrane could lead to a selection of more aggressive clones of tumor cells without Hsp60 on their surface.

In conclusion, Hsp60 has the potential to become a good target for a novel anti-cancer chaperonotherapy, at least in tumor cells that express this protein on their surface. However, it is crucial to obtain more information about the molecular mechanisms underlying the translocation of Hsp60 to the cell membrane. This is necessary for developing means to amplify outer-membrane localization, selectively, in tumor cells and to ensure that the chaperonin remains accessible to antibodies long enough so they can react with it and trigger cell damage.

\section{CONFLICT OF INTEREST}

The authors confirm that this article content has no conflicts of interest.

\section{ACKNOWLEDGMENTS}

This work was partially supported by UniPA (fund "ex-60\%" 2007) and by the Istituto Euro-Mediterraneo di Scienza e Tecnologia (IEMEST), Palermo, Italy (FC and AJLM).

\section{REFERENCES}

[1] Hartl FU, Bracher A, Hayer-Hartl M. Molecular chaperones in protein folding and proteostasis. Nature 2011; 475: 324-32.

[2] Krøll J. The molecular chaperones and the phenomena of cellular immortalization and apoptosis in vitro. Ann N Y Acad Sci 2004; 1019: 568-71.

[3] Nakamoto H, Vígh L. The small heat shock proteins and their clients. Cell Mol Life Sci 2007; 64: 294-306.

[4] Haak J, Kregel KC. 1962-2007: a cell stress odyssey. Novartis Found Symp 2008; 291:3-15; discussion 15-22, 137-40.

[5] Asea A. Hsp70: a chaperokine. Novartis Found Symp 2008; 291:173-9; discussion 179-83, 221-4.

[6] Csermely P, Korcsmáros T, Kovács IA, et al. System biology of molecular chaperone networks. Novartis Found Symp 2008; 291:45-54; discussion 54-8, 137-40.

[7] Pockley AG, Multhoff G. Cell stress proteins in extracellular fluids: friend or foe? Novartis Found Symp 2008; 291: 86-95; discussion 96-100, 137-40.

[8] Quintana FJ, Cohen IR. HSP60 speaks to the immune system in many voices. Novartis Found Symp 2008; 291:101-11; discussion 111-4, 137-40.

[9] Macario AJL, Conway de Macario E. Sick chaperones, cellular stress, and disease. N Engl J Med 2005; 353: 1489-501.

[10] Macario AJL, Conway de Macario E. Chaperonopathies by defect, excess, or mistake. Ann N Y Acad Sci 2007; 1113: 178-91.

[11] Macario AJL, Conway de Macario E. Chaperonopathies and chaperonotherapy. FEBS Lett 2007; 581: 3681-8.

[12] Almeida MB, do Nascimento JL, Herculano AM, et al. Molecular chaperones: toward new therapeutic tools. Biomed Pharmacother 2011; 65: 239-43.

[13] Kampinga HH, Hageman J, Vos MJ, et al. Guidelines for the nomenclature of the human heat shock proteins. Cell Stress Chaperones 2009; 14: 105-11.

[14] Gomez-Monterrey I, Sala M, Musella S, et al. Heat Shock Protein 90 Inhibitors as Therapeutic Agents. Recent Pat Anticancer Drug Discov. 2012 [Epub ahead of print] Feb 9.
[15] Rothbard JB, Kurnellas MP, Brownell S, et al. Therapeutic effects of systemic administration of the chaperone alpha B crystallin associated with binding proinflammatory plasma proteins. J Biol Chem 2012 [Epub ahead of print] Feb 3.

[16] Kang BH. TRAP1 regulation of mitochondrial life or death decision in cancer cells and mitochondria-targeted TRAP1 inhibitors. BMB Rep 2012; 45: 1-6.

[17] da Silva VC, Ramos CH. The network interaction of the human cytosolic 90kDa heat shock protein Hsp90: A target for cancer therapeutics. J Proteomics 2012 [Epub ahead of print] Jan 2.

[18] Guttmann DM, Koumenis C. Source The heat shock proteins as targets for radiosensitization and chemosensitization in cancer. Cancer Biol Ther 2011; 12(12).

[19] Ebrahimi-Fakhari D, Wahlster L, McLean PJ. Molecular chaperones in Parkinson's Disease - present and future. J Parkinsons Dis 2011; 1: 299-320.

[20] Kalia SK, Kalia LV, McLean PJ. Molecular chaperones as rational drug targets for Parkinson's disease therapeutics. CNS Neurol Disord Drug Targets 2010; 9: 741-53.

[21] Tan MH, Smith AJ, Pawlyk B, et al. Gene therapy for retinitis pigmentosa and Leber congenital amaurosis caused by defects in AIPL1: effective rescue of mouse models of partial and complete Aipl1 deficiency using AAV2/2 and AAV2/8 vectors. Hum Mol Genet 2009; 18: 2099-114.

[22] Sigler PB, Xu Z, Rye HS, et al. Structure and function in GroELmediated protein folding. Annu Rev Biochem 1998; 67: 581-608.

[23] Török Z, Horváth I, Goloubinoff P, et al. Evidence for a lipochaperonin: association of active protein-folding GroESL oligomers with lipids can stabilize membranes under heat shock conditions. Proc Natl Acad Sci USA 1997 94: 2192-7.

[24] Macario AJL, Lange M, Ahring BK, et al. Stress genes and proteins in the archaea. Microbiol Mol Biol Rev 1999; 63: 923-67.

[25] Mukherjee K, Conway de Macario E, Macario AJL, et al. Chaperonin genes on the rise: new divergent classes and intense duplication in human and other vertebrate genomes. BMC Evol Biol 2010, 10:64.

[26] Corrao S, Campanella C, Anzalone R, et al. Human Hsp10 and Early Pregnancy Factor (EPF) and their relationship and involvement in cancer and immunity: current knowledge and perspectives. Life Sci 2010; 86: 145-52.

[27] Dencher NA, Frenzel M, Reifschneider NH, et al. Proteome alterations in rat mitochondria caused by aging. Ann N Y Acad Sci 2007; 1100:291-8.

[28] Folli F, Guzzi V, Perego L, et al. Proteomics reveals novel oxidative and glycolytic mechanisms in type 1 diabetic patients' skin which are normalized by kidney-pancreas transplantation. PLoS One 2010;5:e9923.

[29] Cappello F, Conway de Macario E, Marasa L, et al. Hsp60 expression, new locations, functions and perspectives for cancer diagnosis and therapy. Cancer Biol Ther 2008; 7:801-9.

[30] Kaufman BA, Kolesar JE, Perlman PS, et al. A function for the mitochondrial chaperonin Hsp60 in the structure and transmission of mitochondrial DNA nucleoids in Saccharomyces cerevisiae. J Cell Biol 2003;163: 457-61.

[31] Chandra D, Choy G, Tang DG. Cytosolic accumulation of HSP60 during apoptosis with or without apparent mitochondrial release: evidence that its pro-apoptotic or pro-survival functions involve differential interactions with caspase-3. J Biol Chem 2007; 282: 31289-301.

[32] Campanella C, Bucchieri F, Ardizzone NM, et al. Upon oxidative stress, the antiapoptotic Hsp60/procaspase-3 complex persists in mucoepidermoid carcinoma cells. Eur J Histochem 2008; 52: 2218.

[33] Merendino AM, Bucchieri F, Campanella C, et al. Hsp60 is actively secreted by human tumor cells. PLoS One 2010;5:e9247.

[34] Bobrie A, Colombo M, Raposo G, et al. Exosome secretion: molecular mechanisms and roles in immune responses. Traffic 2011; 12: $1659-68$.

[35] Cappello F, Conway de Macario E, Di Felice V, et al. Chlamydia trachomatis infection and anti-Hsp60 immunity: the two sides of the coin. PLoS Pathog 2009;5:e1000552.

[36] Macario AJL, Cappello F, Zummo G, et al. Chaperonopathies of senescence and the scrambling of interactions between the chaperoning and the immune systems. Ann N Y Acad Sci 2010; 1197: 8593. 
[37] Rizzo M, Macario AJL, Conway de Macario E, et al. Heat shock protein-60 and risk for cardiovascular disease. Curr Pharm Des 2011; 17: 3662-8.

[38] Pockley AG, Bulmer J, Hanks BM, et al. Identification of human heat shock protein 60 (Hsp60) and anti-Hsp60 antibodies in the peripheral circulation of normal individuals. Cell Stress Chaperones 1999; 4: 29-35.

[39] Alard JE, Dueymes M, Youinou P, et al. Modulation of endothelial cell damages by anti-Hsp60 autoantibodies in systemic autoimmune diseases. Autoimmun Rev 2007; 6: 438-43.

[40] Cappello F, Czarnecka AM, La Rocca G, et al. Hsp60 and Hsp10 as antitumor molecular agents. Cancer Biol Ther 2007;6: 487-9.

[41] Rahlff J, Trusch M, Haag F, et al. Antigen-specificity of oligoclonal abnormal protein bands in multiple myeloma after allogeneic stem cell transplantation. Cancer Immunol Immunother 2012 [Epub ahead of print] Feb 21.

[42] Cappello F, David S, Peri G, et al. Hsp60: molecular anatomy and role in colorectal cancer diagnosis and treatment. Front Biosci (Schol Ed) 2011; 3: 341-51.

[43] Stangl S, Gehrmann M, Riegger J, et al. Targeting membrane heatshock protein 70 (Hsp70) on tumors by cmHsp70.1 antibody. Proc Natl Acad Sci USA 2011; 108: 733-8.
[44] Cappello F, Conway de Macario E, Zummo G, et al. Immunohistochemistry of human Hsp60 in health and disease: from autoimmunity to cancer. Methods Mol Biol 2011; 787: 245-54.

[45] Zhong H, Yang Y, Ma S, et al. Induction of a tumour-specific CTL response by exosomes isolated from heat-treated malignant ascites of gastric cancer patients. Int J Hyperthermia 2011; 27: 604-11.

[46] Victora GD, Socorro-Silva A, Volsi EC, et al. Immune response to vaccination with DNA-Hsp65 in a phase I clinical trial with head and neck cancer patients. Cancer Gene Ther 2009; 16: 598-608.

[47] Pfister G, Stroh CM, Perschinka H, et al. Detection of HSP60 on the membrane surface of stressed human endothelial cells by atomic force and confocal microscopy. J Cell Sci 2005; 118: 158794.

[48] Lin L, Kim SC, Wang Y, et al. HSP60 in heart failure: abnormal distribution and role in cardiac myocyte apoptosis. Am J Physiol Heart Circ Physiol 2007; 293: H2238-47.

[49] Goh YC, Yap CT, Huang BH, et al. Heat-shock protein 60 translocates to the surface of apoptotic cells and differentiated megakaryocytes and stimulates phagocytosis. Cell Mol Life Sci 2011; 68: 1581-92.

[50] Soltys BJ, Gupta RS. Immunoelectron microscopic localization of the 60-kDa heat shock chaperonin protein (Hsp60) in mammalian cells. Exp Cell Res 1996; 222:16-27. 